\title{
PREFERÊNCIA PARA OVIPOSIÇÃO E CICLO DE VIDA DE MOSCA-NEGRA- DOS-CITROS Aleurocanthus woglumi ASHBY EM ESPÉCIES FRUTÍFERAS ${ }^{1}$
}

\author{
GISLANE DA SILVA LOPES ${ }^{2}$, RAIMUNDA NONATA SANTOS DE LEMOS ${ }^{3}$, \\ JOSÉ RIBAMAR GUSMÃO ARAUJO ${ }^{3}$, LUIZ JUNIOR PEREIRA MARQUES ${ }^{2}$, \\ DANIELE LAVRA VIEIRA ${ }^{2}$
}

RESUMO-Aleurocanthus woglumi Ashby conhecida popularmente como mosca-negra-dos-citros é considerada praga quarentenária A2 no Brasil e ocasiona prejuízo em diversas frutíferas, principalmente em citros (laranja, limão e tangerina). Poucas são as pesquisas relacionadas aos seus aspectos biológicos nas condições ambientais brasileiras. Nesse sentido, o objetivo da pesquisa foi verificar a preferência de oviposição e a duração do ciclo de vida de $A$. woglumi em diferentes hospedeiros. A pesquisa foi conduzida em casa de vegetação, durante o período de março de 2009 a março de 2010. Foram realizados testes de preferência sem chance de escolha em seis hospedeiros, simultaneamente, em períodos de 48 e 72 horas, além da biologia comparada em mangueira e laranjeira. Foram observados nos testes que A. woglumi apresenta preferência por ovipositar nas espécies cítricas (limoeiro, laranjeira e tangerineira), mantendo um padrão de não preferência em cajueiro e goiabeira. Os hospedeiros laranjeira e mangueira não interferiram no ciclo biológico da praga.

Termos para indexação: Aleyrodidae. Biologia. Resistência. Hospedeiro alternativo.

\section{OVIPOSITION PREFERENCE AND LIFE CYCLE OF CITRUS BLACKFLY Aleurocanthus woglumi ASHBY ON FRUIT CROPS}

\begin{abstract}
Aleurocanthus woglumi Ashby, popularly known as the citrus blackfly is considered A2 quarantine pest in Brazil and causes damage in many fruit crops, especially citrus (orange, lemon and tangerine). Few researches related to biological aspects are carried out in Brazilian environmental conditions. The present research aimed to determine the oviposition preference and duration of the life cycle of $A$. woglumi on different hosts. The research was carried out in a greenhouse during the period of March 2009 to March 2010. Tests of preference were done with no chance of choice in six hosts, in periods of 48 and 72 hours, beyond the comparative biology in mango and orange trees. A. woglumi showed preference for laying eggs on citrus species (lemon, orange and mandarin), maintaining a pattern of non-preference in cashew and guava trees. The orange and mango hosts did not interfere in the life cycle of the pest.
\end{abstract}

Index terms: Aleyrodidae, Biology, Resistence, Alternative host.

\section{INTRODUÇÃO}

O Brasil tem-se destacado no mercado internacional com exportações de diversos produtos, como soja, café, laranja, açúcar e álcool. Na atividade citrícola, a colheita concluída em fevereiro de 2010 indicou uma produção em torno de 290 milhões de caixas $40,8 \mathrm{~kg}$, entretanto o volume da safra ficou $20 \%$ abaixo do potencial da região produtora de São Paulo e Minas Gerais, provavelmente por fatores econômicos e fitossanitários (AGRIANUAL, 2011).
A mosca-negra-dos-citros, Aleurocanthus woglumi Ashby, 1915 (Hemiptera: Aleyrodidae), infesta mais de 300 plantas hospedeiras, mas as plantas cítricas constituem-se em hospedeiros mais apropriados para o desenvolvimento de grandes populações (NGUYEN; HAMON, 1993). Além disso, igualmente a mosca-branca (Bemisia tabaci Gennadius, 1889 biótipo B) causa danos diretos por sugar a seiva das folhas e, indiretos, por excretar quantidades abundantes de gotas açucaradas, que cobrem superfícies de folhas e frutos (EPPO,

'(Trabalho 032-12). Recebido em: 09-08-2012. Aceito para publicação em: 04-01-2012. Parte da dissertação do primeiro autor apresentada ao Programa de Pós-Graduação em Agroecologia/UEMA.

2Programa de Pós-Graduação em Agroecologia/CCA/UEMA. E-mails: gslopes11@yahoo.com.br; d.lavra@hotmail.com; luiz1000x@ yahoo.com.br

${ }^{3}$ Dr. Professores do Deptartamento de Fitotecnia e Fitossanidade/CCA/UEMA- Caixa Postal 9, CEP 65.055-098, São Luís-MA. E-mails: r.lemos@elo.com.br (autor correspondente); gusmão@elo.com.br 
2002), dificultando as trocas gasosas e depreciando o produto.

Atualmente, diversos produtores têm-se preocupado com sua dispersão, anteriormente restrita aos Estados do Pará, Amapá, Amazonas, Tocantins e Maranhão (LEMOS et al., 2006), São Paulo (PENA et al., 2008), Goiás (YAMAMOTO et al., 2008), Paraíba (LOPES et al., 2010), e Roraima (CORREIA et al., 2011), e relatos nos Estados do Ceará, Rio Grande do Norte, Pernambuco e Bahia pelas agências de defesas fitossanitárias.

Por se tratar de uma praga com hábito alimentar polífago, faz-se necessário o conhecimento dos hospedeiros preferenciais, revelando dessa forma aqueles capazes de garantir o desenvolvimento de seu ciclo. De acordo com Thompson (1988), insetos polífagos ovipositam em vários hospedeiros diferentes, sendo comum as fêmeas exibirem uma hierarquia de preferência, pois alguns hospedeiros são mais preferidos em relação a outros. $\mathrm{O}$ mesmo autor também reportou que a idade, porte e estágio reprodutivo das plantas e das condições de nutrientes em que as plantas cresceram, podem interferir na preferência e no desempenho de alguns insetos.

Dowell et al. (1978) avaliaram o desenvolvimento de $A$. woglumi em seis hospedeiros naturais, atestando que o limão proporcionou maior sobrevivência ao inseto. De maneira similar, Fasulo e Brooks (1993) observaram que os citros e a manga foram hospedeiros preferidos, e o pomelo, o menos desejável das variedades de citros. No Pará, as plantas cítricas, mangueiras e grumixamas são hospedeiras da praga (CUNHA, 2003). Em São Paulo, Raga e Costa (2008) relataram infestações em laranjeiras, abacateiro, goiabeira, bananeira e caquizeiro. Ronchi-Teles et al. (2009), no Amazonas, reportaram o inseto atacando citros, mangueiras $\mathrm{e}$ café, e no Maranhão houve registros em mangueira e citros (LEMOS et al., 2006).

Elizondo e Quezada (1990) observaram em pomares citrícolas na Costa Rica que a duração do ciclo de vida de $A$. woglumi, em temperatura de $16^{\circ} \mathrm{C}$, foi de 567 dias; no entanto, a $27^{\circ} \mathrm{C}$, o ciclo ocorreu em 74 dias, ou seja, a temperatura influenciou diretamente em seu desenvolvimento e na fecundidade. No estado da Flórida, o ciclo de vida de ovo até adulto pode levar de 45 a 133 dias, dependendo da temperatura (NGUYEN et al., 1998). Na Venezuela, Boscán (2001) observou ciclo total variando de 54 a 103 dias.

Cunha (2003) demonstrou que a duração do ciclo de ovo a adulto variou de 59 a 88 dias no Estado do Pará, confirmando as condições térmicas como as principais responsáveis pela duração do ciclo de
A. woglumi. Segundo Ronchi-Telles et al. (2009), no Estado do Amazonas, o ciclo médio foi de 71,76 $\pm 2,07$ dias, caracterizando-a como uma espécie multivoltina. Segundo Thompson (1988), além das condições climáticas, fatores externos, como predação e parasitismo, também podem modificar a biologia de insetos fitófagos.

Assim, estudos da relação inseto-planta podem contribuir para um melhor conhecimento sobre os critérios de aceitação e preferência de plantas hospedeiras, auxiliando no controle e manejo, principalmente em pequenas propriedades no Maranhão, onde a citricultura é promissora através dos cultivos de tangerina e limas-ácidas. Dessa forma, a pesquisa objetivou avaliar a preferência de oviposição e a duração do ciclo de vida de $A$. woglumi em diferentes hospedeiros, fornecendo, assim, mecanismos para implantação de programas de MIP, que sejam menos agressivos ao ambiente.

\section{MATERIAL E MÉTODOS}

\section{Testes de preferência de oviposição de Aleurocanthus woglumi}

O experimento foi conduzido em casa de vegetação, localizada na Fazenda-Escola de São Luís (São Luís-MA) Câmpus da Universidade Estadual do Maranhão - UEMA, no período de março de 2009 a março de 2010. Os adultos de A. woglumi utilizados nos testes de preferência de oviposição foram coletados em pomar de lima-ácida Tahiti (Citrus latifolia Tanaka), localizado no município de São José de Ribamar-MA, em área de 3,5 ha, com espaçamento de 7,0 x 7,0 m. Esta área experimental está representada na carta topográfica SA $23 \mathrm{com}$ as seguintes coordenadas planas 9.713.315,615 $\mathrm{S}$ e 875.44,986 WGR (IBGE, 2003).

Foram avaliados brotos de seis plantas hospedeiras, simultaneamente, em testes sem chance de escolha das seguintes espécies e variedades: manga (Rosa), lima-ácida (Tahiti), tangerina (Ponkan), laranja (Rubi), caju (Comum 18) e goiaba (Pêra). O material vegetal foi previamente lavado com detergente neutro diluído em água $(70 \%)$ e, posteriormente, em água corrente.

Para os testes de oviposição sem chance de escolha, foram confeccionadas 30 gaiolas cilíndricas em arame recozido, nas dimensões de $16,5 \mathrm{~cm}$ de raio e $55 \mathrm{~cm}$ de altura, cobertas com tecido voile. Para a fixação das gaiolas nos vasos, utilizaram-se dez centímetros de arame recozido da própria estrutura, ficando disponíveis $45 \mathrm{~cm}$ para o voo dos insetos. $\mathrm{Na}$ parte interior e central de cada vaso, colocou- 
se um tubo plástico transparente com capacidade para $70 \mathrm{~mL}$ contendo água à metade e um chumaço de algodão para evitar a desidratação do material vegetal. Em seguida,foram colocadas oito folhas novas (brotações) de cada um dos hospedeiros selecionados para a realização dos testes.

Utilizando-se de recipientes de sucção (tubos de ensaio), coletaram-se 3.500 insetos, para garantir uma infestação satisfatória das plantas hospedeiras. Devido à intensa movimentação dos insetos, não foi possível realizar a sexagem dos adultos. Após a captura, foram liberados aproximadamente 100 adultos em cada gaiola, onde permaneceram para oviposição por 48 horas no primeiro teste e por 72 horas no segundo. Decorrido os períodos de infestação, os insetos foram liberados em campo e contou-se o número de postura/planta e o número de ovos/postura. Nos dois testes de preferência de oviposição, adotou-se o delineamento estatístico inteiramente casualizado, com seis tratamentos (variedades) e cinco repetições. Após a contagem do número de ovos/ brotação, calculou-se o índice de preferência para oviposição, utilizando-se da fórmula IPO $=[(\mathrm{A}-\mathrm{B}) /(\mathrm{A}+\mathrm{B})]$ x 100, proposta por Fenemore (1980), em que: $A=n^{\circ}$ de ovos contados no genótipo avaliado, e $\mathrm{B}=$ número de ovos contados no genótipo-padrão. $\mathrm{O}$ índice varia de +100 para muito estimulante; 0 (zero) para neutro, e -100 para total deterrência. O hospedeiro lima-ácida foi escolhido como padrão de preferência, partindo-se do princípio de Hopikns, de preferência prévia por lima-ácida Tahiti.

A classificação dos materiais (estimulante/ deterrente) foi feita a partir da comparação das médias de ovos dos tratamentos, com a média do genótipo-padrão, considerando-se o erro-padrão para diferenciação dos mesmos. Sendo estimulante, valor positivo e maior que o erro-padrão; neutro, valor positivo ou negativo e menor que o erro-padrão; deterrente, valor negativo e maior que o erropadrão (COSTA et al., 2004). A análise estatística foi realizada por meio de ANOVAs para medidas repetidas, usando-se o programa Statistica 7.0 (STATSOFT 1984-2004), seguido de teste de média (Fisher LSD a 5\% de probabilidade).

\section{woglumi}

Duração do ciclo de vida de Aleurocanthus

O experimento foi conduzido em casa de vegetação na Fazenda - Escola de São Luís, no período de 12 de janeiro a 29 de março de 2010. Foram avaliados três hospedeiros, mediante preferência relacionada em literatura e em testes preliminares de oviposição, das seguintes variedades enxertadas: laranjeira Pera (Citrus sinensis L. Osbeck), com 8 meses de idade; mangueira Haden (Mangifera indica L.) e cajueiro-anão clone CCP-76 (Anacardium occidentale L.), ambos com 9 meses de idade. Estas mudas foram previamente lavadas com detergente neutro diluído $(70 \%)$ e, posteriormente, em água corrente. Após assepsia, foram cobertas com tecido voile, preso por ligas elásticas no caule de cada planta.

Para infestação das plantas hospedeiras, foram capturados 540 insetos adultos, provenientes de mudas de laranjeiras infestadas do próprio viveiro da Fazenda-Escola de São Luís, utilizandose de recipientes de sucção. Devido à intensa movimentação dos insetos, não foi possível realizar a sexagem dos adultos. Foram liberados 20 adultos em cada planta, mantendo-os por 72 horas para a realização da postura. Em seguida, esses adultos foram retirados e procedeu-se à marcação, contagem do número de posturas e número de ovos/postura colocados nas folhas das plantas em estudo.

As avaliações foram realizadas diariamente, observando-se as seguintes variáveis: período de incubação dos ovos (dias), duração dos instares (dias), longevidade de adultos (dias), ciclo total (ovo a adulto) e viabilidade (\%) de todas as fases. Com relação à análise estatística, optou-se pela não paramétrica devido aos diferentes números de repetição. $O$ teste não paramétrico adotado foi o de Wilcoxon, a $5 \%$ de probabilidade $(\mathrm{p}<0,05)$, realizado pelo programa SAS System, versão 8.2 (SAS, 2001).

\section{RESULTADOS E DISCUSSÃO}

\section{Testes de preferência de oviposição}

Os adultos de $A$. woglumi foram confinados em testes sem chance de escolha, avaliando-se a oviposição nos hospedeiros, separadamente, em períodos de 48 e 72 horas. A realização destes testes serviu como indicativo se haveria ou não algum tipo de resistência e se existiria estabilidade desse comportamento.

Quando os hospedeiros foram expostos à oviposição de $A$. woglumi por um período de 48 horas de forma isolada, verificou-se que as brotações de laranjeira e limoeiro foram preferidas em relação aos demais hospedeiros testados, com 153,6 e 134,6 ovos/broto, respectivamente. Tangerineira apresentou a média de 47,4 ovos/broto, mas não diferiu das brotações da mangueira com 1,2 ovo/ broto, goiabeira com 8,6 ovos/broto e cajueiro sem 
oviposição (Figura 1).

De acordo com Fasulo e Brooks (1993), a preferência pode variar dentro das variedades de citros como também as fêmeas podem basear sua oviposição na melhor escolha para o sucesso da prole, pois isto representaria uma vantagem, visto que poderiam escolher os hospedeiros de melhor qualidade.

No teste sem chance de escolha, em que o tempo de permanência de $A$. woglumi nas gaiolas foi de 72 horas, brotos de laranjeira apresentaram maior preferência de oviposição ( 800,2 ovos/broto), diferindo-se dos demais hospedeiros (Figura 1). O número de ovos depositados nos hospedeiros lima-ácida, tangerina e manga não diferiram entre si (311,2; 177 e 42,6 ovos/broto, respectivamente), e o cajueiro apresentou deterrência, pois os insetos não ovipositaram em nenhuma das brotações. Esses resultados diferem de Pena et al. (2009), que verificaram a preferência por lima-ácida, demonstrando que o comportamento desses aleirodídeos podem manter- se para alguns parâmetros ou modificar-se, dependendo, principalmente, das condições ambientais a que estão expostos.

Caso semelhante foi verificado por Alves et al. (2005), que avaliaram a preferência para oviposição de $B$. tabaci em genótipos de aboboreira e constataram que as variedades Novita, Caserta e Exposição apresentaram resistência do tipo não preferência, pois mesmo quando o inseto não tinha outro genótipo para ovipositar, os valores encontrados foram baixos nestes materiais.

Com base nos resultados obtidos, foi calculado o IPO - Índice de Preferência de Oviposição (Tabela 1). A preferência de oviposição nos testes sem chance de escolha ficou na espécie cítrica (laranja), que foi considerada neutra (48 horas) e estimulante (72 horas). Foi verificada característica deterrente da goiabeira, mangueira e cajueiro, em ambos os períodos avaliados. A deterrência de determinado material indica um potencial de resistência que deve ser estudado e melhorado para uso em programas de manejo integrado de pragas, conforme Baldin et al. (2000) e Oriani et al., (2005). Este resultado difere de alguns autores que reportaram a utilização de tais frutíferas pelo aleirodídeo (PENA et al., 2008, 2009; RAGA; COSTA, 2008; RONCHI-TELES et al., 2009). Contudo, este comportamento pode ser explicado, uma vez que este índice é calculado em condições de confinamento dos diversos hospedeiros, inclusive aquele padrão, no qual os valores das oviposições são bem elevados, pois o inseto é submetido às condições e hospedeiro adequados.

Outro ponto relevante na pesquisa foi observado no IPO do cajueiro, que no teste sem chance de escolha e em diferentes períodos (48 e 72 horas) atingiu valor de deterrência, com picos máximos de inibição, ou seja, manteve estável sua resistência tipo não preferência mesmo quando o inseto não detinha nenhum outro material vegetal para ovipositar.

\section{Ciclo de Vida de Aleurocanthus woglumi}

O desenvolvimento do ciclo de $A$. woglumi foi avaliado apenas em mudas de laranjeira e mangueira, uma vez que não houve oviposição nas plantas de cajueiro mesmo mantendo os adultos confinados por período de 72 horas e apesar dos relatos em literatura (NGUYEN; HAMON, 1993; BOSCÁN, 2001; BRASIL, 2007).

Para a obtenção do ciclo biológico, foram observados 68 ovos em laranjeira e 156 ovos em mangueira. Pelos resultados, observou-se que não houve diferença significativa no período de incubação dos ovos para os dois hospedeiros (Tabela 2). Estes valores foram próximos dos encontrados por Dowell et al., (1981), os quais tiveram duração entre 7 a 10 dias para as condições da Flórida, e por Boscán (2001), que registrou, na Venezuela, tempo de eclosão variando de 10 a 18 dias em laboratório e de 12 a 26 dias sob condições de campo. Estes valores diferiram dos encontrados por Ronchi-Telles et al., (2009), em que o período embrionário para as condições do Estado do Amazonas foi de 14,43 $\pm 0,75$ dias.

$\mathrm{Na}$ fase de ninfa de $1^{\circ}$ e $2^{\circ}$ instares também não houve diferença significativa entre os hospedeiros testados, sendo a duração do $1^{\circ}$ instar entre 7,85 e 9,52 dias e entre 6,94 e 7,36 dias para o $2^{\circ}$ instar, corroborando os resultados de Pena et al. (2009). A duração do $1^{\circ}$ e $2^{\circ}$ instares também esteve dentro dos valores obtidos por Boscán (2001), que encontrou média de 6 a 19 dias para o $1^{\circ}$ instar e entre 6 e 7 dias para o $2^{\circ}$ instar, em condições de campo, na Venezuela. Entretanto, apesar de a média (dias) ter sido similar entre as pesquisas, as condições maranhenses propiciam valores sempre inferiores, que possibilitam uma redução maior ao final do ciclo do inseto.

Os períodos correspondentes a $3^{\circ}$ e $4^{\circ}$ instares apresentaram significância em comparação aos dois hospedeiros utilizados, tendo uma duração maior na laranjeira referente ao $3^{\circ}$ instar e na mangueira em relação ao $4^{\circ}$ instar. Todavia, ambos os valores estão incluídos dentro de uma faixa encontrada em outros trabalhos que demonstraram um período de $3^{\circ}$ instar variando entre 6 e 20 dias (NGUYEN 
HAMON, 1993) e de 8 a 14 dias (CUNHA, 2003). Quanto às ninfas de $4^{\circ}$ instar, pesquisas reportaram variação entre 16 e 50 dias (DOWELL et al., 1981) e 33,58 $\pm 2,12$ dias para as condições do Amazonas (RONCHI-TELLES et al., 2009).

Assim como em outras pesquisas, a fase correspondente ao $4^{\circ}$ instar, também definida como pupário, apresentou a maior duração de dias (NGUYEN et al., 1998; BOSCÁN, 2001; PENA et al., 2009). A longevidade dos adultos e a duração do ciclo total (ovo-adulto) não apresentaram diferença significativa entre os hospedeiros estudados.

Segundo Gyeltshen et al. (2005), a duração do ciclo de vida e o número de gerações por ano de Aleurocanthus spiniferus (Aleyrodidae) é muito influenciada pelo clima predominante, caso similar aos resultados deste estudo, pois a duração do ciclo de vida de $A$. woglumi foi inferior aos encontrados por Pena et al. (2009) e Cunha (2003). Assim como nas referidas pesquisas, pode-se inferir que $A$. woglumi é uma espécie multivoltina, pois, de acordo com a duração média do ciclo, é possível que ocorram, em média, cinco gerações ao ano em São Luís (MA).

Além da duração de cada fase do ciclo da mosca-negra, avaliou-se a viabilidade em cada estádio de desenvolvimento nos hospedeiros laranjeira e mangueira, onde não foi observada diferença significativa na viabilidade das fases de $A$. woglumi (Tabela 3). Dados semelhantes foram encontrados por Pena et al. (2009) quando compararam a viabilidade da fase imatura em laranjeira Pera com mangueira e encontraram taxas de 56,59 $\pm 9,68 \%$ e

\section{$36,62 \pm 9,85 \%$, respectivamente.}

Cunha (2003) observou menores percentuais de viabilidade em relação aos encontrados no presente estudo: ovo $(65,4 \%)$, ninfa $1(41,7 \%)$, ninfa $2(26,3 \%)$, ninfa $3(19,2 \%)$ e ninfa $4(13,6 \%)$. Esse autor relacionou a baixa taxa de sobrevivência à ação de predadores e parasitoides.

No estudo do ciclo biológico da moscanegra, tanto a duração como a viabilidade das fases de desenvolvimento foram muito afetadas pelo controle natural, pois nas fases de ovo até ninfa de $2^{\circ}$ instar observou-se grande mortalidade das mesmas devido à presença de agentes externos, como os ácaros Amblyseius acalyphus Denmark \& Muma, 1973 e Amblyseius aerialis Muma, 1955 (Acari: Phytoseiidae) e o aparecimento de fungos, que, mediante a demora de mudança dos instares de $A$. woglumi, acabaram por inviabilizar diversas ninfas e ovos, tornando-os mumificados ou secos.

O conhecimento do ciclo de vida de $A$. woglumi permite o desenvolvimento de estratégias de manejo agroecológico para os agricultores, uma vez que a produção frutífera do Estado é formada basicamente por pequenos produtores que não dispõem de condições financeiras adequadas para controlar grandes infestações da praga. Assim, as características comportamentais do inseto indicam uma suscetibilidade ao controle natural que pode ser fortalecido com práticas de consórcio com espécies deterrentes e preservação de inimigos naturais que ocorrem nos pomares.

TABELA 1 - Índice (erro-padrão) e classificação de preferência para oviposição de Aleurocanthus woglumi em seis hospedeiros, em testes sem chance de escolha. São Luís (MA), 2009.

\begin{tabular}{cccc}
\hline Condição & Hospedeiro & IPO (EP) & Classificação \\
\hline TSCE 48 h & Limão & --- & Padrão \\
& Caju & $-100,00(0,00)$ & Deterrente \\
& Goiaba & $-85,90(14,10)$ & Deterrente \\
& Laranja & $-8,12(33,38)$ & Neutro \\
& Manga & $-97,18(2,82)$ & Deterrente \\
& Tangerina & $-33,80(23,71)$ & Deterrente \\
\hline TSCE 72 h & Limão & --- & Padrão \\
& Caju & $-80,00(20,00)$ & Deterrente \\
& Goiaba & $-57,10(39,37)$ & Deterrente \\
& Laranja & $40,80(18,20)$ & Estimulante \\
& Manga & $-65,52(18,60)$ & Deterrente \\
& Tangerina & $-13,77(30,58)$ & Neutro \\
\hline
\end{tabular}

IPO = Índice de preferência para oviposição $[(\mathrm{A}-\mathrm{B})] /[(\mathrm{A}+\mathrm{B})]$ x 100. A = número de ovos no genótipo avaliado; B = número de ovos no genótipo-padrão; Estimulante = valor positivo e maior que o erro-padrão; neutro = valor positivo ou negativo e menor que o erropadrão; deterrente $=$ valor negativo e maior que o erro-padrão. 
TABELA 2 - Duração média (dias) e erro-padrão das diferentes fases de desenvolvimento de Aleurocanthus woglumi mantidas em mangueira (Mangifera indica) e laranjeira (Citrus sinensis) em casa de vegetação. São Luís (MA), 2010.

\begin{tabular}{|c|c|c|c|c|c|c|c|}
\hline Hospedeiro & $\begin{array}{c}\text { Período de } \\
\text { Incubação (EP) }\end{array}$ & $\begin{array}{c}\text { Ninfa } 1 \\
(E P)^{1}\end{array}$ & $\begin{array}{c}\text { Ninfa } 2 \\
(E P)^{1}\end{array}$ & $\begin{array}{c}\text { Ninfa } 3 \\
(E P)^{1}\end{array}$ & $\begin{array}{c}\text { Ninfa } 4 \\
(E P)^{1}\end{array}$ & $\begin{array}{c}\text { Longevidade } \\
\text { de Adultos (EP) }\end{array}$ & $\begin{array}{l}\text { Ciclo Total } \\
\text { (EP) }^{1}\end{array}$ \\
\hline Laranjeira & $\begin{array}{l}10,25 \\
(0,09)\end{array}$ & $\begin{array}{l}7,85 \\
(0,15)\end{array}$ & $\begin{array}{c}6,94 \\
(0,26)\end{array}$ & $\begin{array}{c}9,53 \\
(0,26)\end{array}$ & $\begin{array}{l}26,98 \\
(0,62)\end{array}$ & $\begin{array}{c}5,02 \\
(0,24)\end{array}$ & $\begin{array}{l}66,71 \\
(0,71)\end{array}$ \\
\hline Mangueira & $\begin{array}{c}9,90 \\
(0,12) \\
\end{array}$ & $\begin{array}{c}9,52 \\
(0,43) \\
\end{array}$ & $\begin{array}{c}7,36 \\
(0,43) \\
\end{array}$ & $\begin{array}{c}7,67 \\
(0,54) \\
\end{array}$ & $\begin{array}{l}28,65 \\
(1,24) \\
\end{array}$ & $\begin{array}{c}4,68 \\
(0,29) \\
\end{array}$ & $\begin{array}{l}69,00 \\
(0,65) \\
\end{array}$ \\
\hline $\operatorname{Pr}<Z$ & $0,2376^{\mathrm{ns}}$ & $0,1358^{\mathrm{ns}}$ & $0,1203^{\mathrm{ns}}$ & $0,0009^{*}$ & $0,0295 *$ & $0,2793^{\mathrm{ns}}$ & $0,0612^{\mathrm{ns}}$ \\
\hline CV (\%) & 12,62 & 40,66 & 32,42 & 28,12 & 16,93 & 30,78 & 6,54 \\
\hline
\end{tabular}

${ }^{1}$ significância da comparação estatística entre médias provada pelo teste não paramétrico de Wilcoxon, a 5\% de probabilidade (p < 0,05).

TABELA 3 - Viabilidade média (\%) e erro-padrão das diferentes fases de desenvolvimento de Aleurocanthus woglumi mantidas em mangueira (Mangifera indica) e laranjeira (Citrus sinensis) em casa de vegetação. São Luís (MA), 2010.

\begin{tabular}{|c|c|c|c|c|c|c|}
\hline \multirow[b]{2}{*}{ Hospedeiro } & \multicolumn{6}{|c|}{ Viabilidade (\%) } \\
\hline & $\begin{array}{l}\text { Ovo } \\
(\mathrm{EP})^{1}\end{array}$ & $\begin{array}{c}\text { Ninfa } 1 \\
(E P)^{1}\end{array}$ & $\begin{array}{c}\text { Ninfa } 2^{2} \\
\text { (EP) }^{1}\end{array}$ & $\begin{array}{c}\text { Ninfa } 3 \\
(E P)^{1}\end{array}$ & $\begin{array}{c}\text { Ninfa } 4^{4} \\
(E P)^{1}\end{array}$ & $\begin{array}{c}\text { Adultos } \\
\text { (EP) }{ }^{1}\end{array}$ \\
\hline Laranjeira & $\begin{array}{l}58,13 \\
(7,52)\end{array}$ & $\begin{array}{l}57,66 \\
(8,52)\end{array}$ & $\begin{array}{l}51,32 \\
(8,88)\end{array}$ & $\begin{array}{l}56,54 \\
(9,20)\end{array}$ & $\begin{array}{l}59,95 \\
(9,53)\end{array}$ & $\begin{array}{l}61,54 \\
(9,73)\end{array}$ \\
\hline Mangueira & $\begin{array}{c}68,59 \\
(11,83)\end{array}$ & $\begin{array}{c}49,93 \\
(17,02)\end{array}$ & $\begin{array}{c}53,53 \\
(18,77)\end{array}$ & $\begin{array}{c}53,97 \\
(17,96)\end{array}$ & $\begin{array}{c}53,33 \\
(21,08)\end{array}$ & $\begin{array}{c}50,00 \\
(22,36)\end{array}$ \\
\hline $\operatorname{Pr}<Z$ & $0,2478^{\text {ns }}$ & $0,3259^{\text {ns }}$ & $0,4700^{\text {ns }}$ & $0,3519^{\text {ns }}$ & $0,5000^{\text {ns }}$ & $0,3148^{\text {ns }}$ \\
\hline CV (\%) & 60,86 & 75,70 & 86,34 & 81,50 & 82,43 & 84,04 \\
\hline
\end{tabular}

${ }^{1}$ significância da comparação estatística entre médias provada pelo teste não paramétrico de Wilcoxon, a 5\% de probabilidade. Dados transformados em arc-sen $\sqrt{ } \mathrm{x} / 100$.

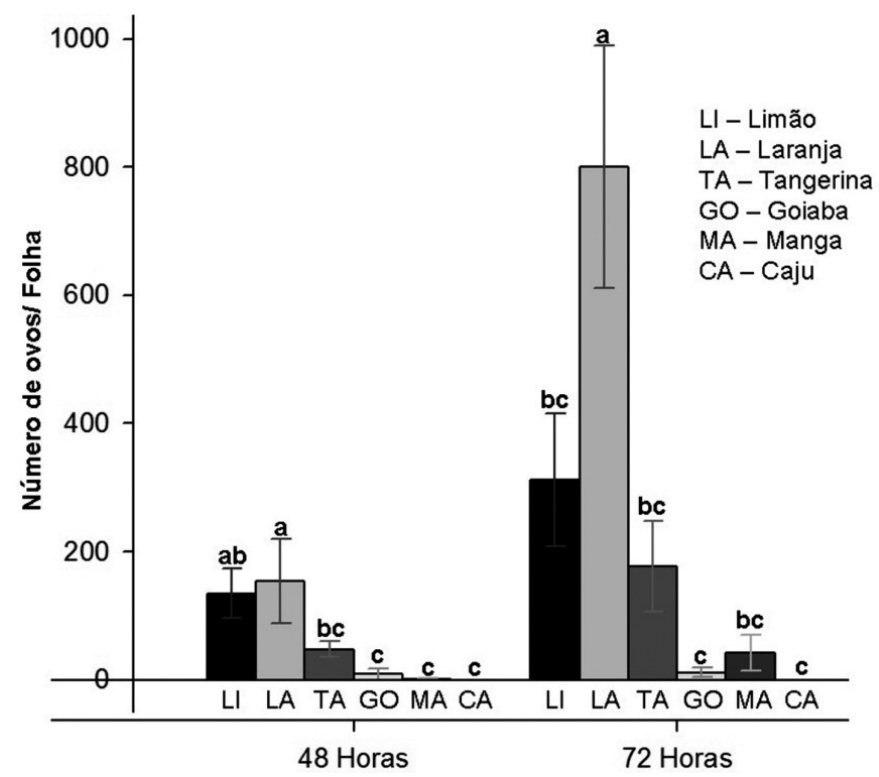

FIGURA 1 - Número de ovos de Aleurocanthus woglumi por folha de diferentes hospedeiros após 48 e 72 horas de exposição, em casa de vegetação, em teste sem chance de escolha. São Luís (MA), 2009.

Médias seguidas da mesma letra não diferem significativamente entre si, pelo teste de Fisher, a 5\% de probabilidade. 


\section{CONCLUSÕES}

1 - Aleurocanthus woglumi apresenta preferência por ovipositar em espécies cítricas e mantem um padrão de não preferência em cajueiro e goiabeira.

2 - O ciclo biológico de $A$. woglumi mostra semelhante em laranjeira e mangueira.

\section{AGRADECIMENTOS}

Os autores agradecem à Fundação de Amparo à Pesquisa e ao Desenvolvimento Tecnológico do Estado do Maranhão - FAPEMA, pelo apoio financeiro a este projeto.

\section{REFERÊNCIAS}

AGRIANUAL: anuário da agricultura brasileira. 16.ed. São Paulo: FNP, Consultoria e Agroinformação, 2011. 482p.

ALVES, A. C.; LOURENÇÃO, A. L.; MELO, A. M. T. Resistência de Genótipos de Aboboreira a Bemisia tabaci (Genn.) biótipo B (Hemiptera: Aleyrodidae). Neotropical Entomology, Londrina, v. 34, n.6, p.973-979, 2005.

BALDIN, E. L. L., TOSCANO, L. C.; LIMA, A. C. S.; LARA, F. M.; BOIÇA JR., A. L. Preferência para oviposição de Bemisia tabaci biótipo "B" por genótipos de Cucurbita moschata e Cucurbita maxima. Boletín de Sanidad Vegetal Plagas, Madrid, v. 26, p. 409-413, 2000.

BOSCÁN, N. La mosca prieta de los cítricos Aleurocanthus woglumi Ashby (Homoptera: Aleyrodidae). In: OJASTI, J. (Org.). Estudio sobre el estado actual de las especies exóticas: proyecto estrategia regional de biodiversidad para los países del trópico andino. Caracas: Comunidad Andina/ Banco Interamericano de Desarrollo, 2001. 220p.

BRASIL. Ministério da Agricultura, Pecuária e Abastecimento. Secretaria de Defesa Agropecuária. Instrução normativa $\mathrm{n}^{\circ} 52$, de 20 de novembro de 2007. Diário Oficial da República Federativa do Brasil, Poder Judiciário, Brasília, DF, 21 de nov. 2007. Seção 1, p.31.
CORREIA, R. G.; LIMA, A. C. S.; FARIAS, P. R. S.; MACIEL, F. C. S.; SILVA, M. W.; SILVA, A. G. Primeiro registro da ocorrência de mosca-negrados-citros, Aleurocanthus woglumi Ashby, 1915 (Hemiptera: Aleyrodidae) em Roraima. Revista Agro@mbiente On-line, Boa Vista, v.5, n.3, p. 245-248, 2011.

COSTA, N. P.; SANTOS, T. M.; BOIÇA JÚNIOR, A. L. Preferência para oviposição de Bemisia tabaci biótipo-B em genótipos de caupi. Acta Scientiarum Agronomy, Maringá, v. 26, n. 2, p. 227-230, 2004.

CUNHA, M. L.A. Distribuição geográfica, aspectos biológicos e controle químico da mosca negra dos citros, Aleurocanthus woglumi Ashby (Hemiptera: Aleyrodidae), nas condições ambientais do Estado do Pará. Belém. 2003. 57 f. Dissertação (Mestrado em Agronomia) - Universidade Federal Rural da Amazônia, Belém, 2003.

DOWELL, R.V.; CHERRY, R. H.; FITZPATRICK, G. E.; REINERT, J. A.; KNAPP, J. L. Biology, plantinsect relations, and control of the citrus blackfly. Florida Agricultural Experiment Station Bulletin, Gainesville, n. 818, p.1-48, 1981.

DOWELL, R. V.; REINERT, J. A.; FITZPATRICK, G. E. Development and survivoship of the citrus blackfly Aleurocanthus woglumi on six citrus hosts. Environmental Entomology, Maryland, v. 7, n. 4, p. $524-525,1978$.

ELIZONDO, J. M.; QUEZADA, J. R. Identificación y evalución de los enemigos naturales de la mosca prieta de los cítricos Aleurocanthus woglumi Ashby (Homoptera: Aleyrodidae) en cuatro zonas citrícolas de Costa Rica. Revista Interamericana de Ciencias Agrícolas, Turrialba, v. 40, n. 2, p. 190-197, 1990.

EPPO- European Plant Protection Organization. Diagnostic protocols for regulated pests. EPPO Bulletin, v. 32, n. 2, p. 261-265, 2002.

FASULO, T. R.; BROOKS, R. F. Whitefly pests of Florida citrus. EENY, Gainesville, n.815, 1993. Disponível em: $<$ http://edis.ifas.ufl.edu/ $>$. Acesso: 21 jan 2010.

FENEMORE, P. G. Oviposition of potato tuber moth, Phthorimaea operculella Zell. (Lepidoptera: Gelechiidae) identification of host-plant factors influencing oviposion response. New Zealand Journal of Zoology, Wellington, v. 7, n.3, p. 435439, 1980. 
GYELTSHEN, J.; HODGES, A.; HODGES, G. S. Orange spiny whitefly, Aleurocanthus spiniferus Quaintance (Insecta: Hemiptera: Aleyrodidae). EENY, Gainesville, n. 341, 2005. Disponível em: $<\underline{\text { http://edis.ifas.ufl.edu/pdffiles/IN/IN61800.pdf }>}$ Acesso em: 10 fev. 2010.

IBGE - Instituto Brasileiro de Geografia e Estatística. Rede brasileira de monitoramento contínuo. 2003. Disponível em: $<$ http://www.sidra.ibge.gov.br/bda/ agri/40default.asp>. Acesso em: 20 jan. 2008.

LEMOS, R. N. S. de; SILVA, G. S.; ARAÚJO, J. R. G.; CHAGAS, E. F.; MOREIRA, A. A.; SOARES, A. T. M. Ocorrência de Aleurocanthus woglumi Ashby (Hemiptera: Aleyrodidae) no Maranhão. Neotropical Entomology, Londrina, v. 35, n. 4, p. 558-559, 2006.

LOPES, E. B.; BRITO, C. H.; BATISTA, J. L.; SILVA, A. B. Ocorrência da mosca-negra-dos-citros (Aleurocanthus woglumi) na Paraíba. Tecnologia \& Ciência Agropecuária, João Pessoa, v.4, n.1, p.19-22, 2010.

NGUYEN, R.; HAMON, A. B. Citrus blackfly, Aleurocanthus woglumi Ashby (Homoptera, Aleyrodidae). DPI Entomology Circular, Gainesville, n. 360, 1993. Disponível em: $<\underline{\text { http:// }}$ edis.ifas.ufl.edu/CH114> . Acesso em: 09 maio 2009.

NGUYEN, R.; HAMON, A. B.; FASULO, T. R. Citrus blackfly, Aleurocanthus woglumi Ashby (Insecta: Hemiptera: Aleyrodidae). EENY, Gainsville, n. 42, 1998. Disponível em: $<\underline{\text { http://edis.ifas.ufl.edu/ }}$ IN199>. Acesso em: 09 maio 2009.

ORIANI, M. A. G.; VENDRAMIN, J. D.; BRUNHEROTO, R. Atratividade e não- preferência para oviposição de Bemisia tabaci (Genn.) biótipo B (Hemiptera: Aleyrodidae) em genótipos de feijoeiro. Neotropical Entomology, Londrina, v. 34, n. 1, p. 105-111, 2005.

PENA, M. R.; SILVA, N. M.; VENDRAMIM, J. D.; LOURENÇÃO, A. L.; HADDAD, M. D.L. Biologia da mosca-negra-dos-citros, Aleurocanthus woglumi Ashby (Hemiptera: Aleyrodidae), em três plantas hospedeiras. Neotropical Entomology, Londrina, v.38, n.2, p.254-261, 2009.
PENA, M. R.; VENDRAMIN, J. D.; LOURENÇÃO, A. L.; SILVA, N. M.; YAMAMOTO, P. T.; GONÇALVES, M. S. Ocorrência da moscanegra-dos-citros, Aleurocanthus woglumi Ashby (Hemiptera: Aleyrodidae) no Estado de São Paulo. Revista Agricultura, Piracicaba, v. 83, p. 61-65, 2008

RAGA, A.; COSTA, V. A. Mosca negra dos citros. São Paulo: Agência Paulista de Tecnologia dos Agronegócios/ Instituto Biológico. 2008. p.1-9 (Documento Técnico, 001). Disponível em: $<\underline{\text { http:// }}$ www.biologico.sp.gov.br/docs/dt/mosca negra.pdf $>$ Acesso em: 09 maio 2009.

RONCHI-TELES, B.; PENA, M. R.; SILVA, N. M. Observações sobre a ocorrência de Mosca-Negrados-Citros, Aleurocanthus woglumi Ashby, 1915 (Hemiptera: Aleyrodidae) no estado do Amazonas. Acta Amazônica, Manaus, v. 39, n. 1, p. 241-244, 2009.

SAS System. Version 8.2. Cary: SAS Institute, 2001. 6 CD-ROM. Windows 98.

STATSOFT. Statistica: data analysis software system. Version 7.0. Tulsa, 2004.

THOMPSON, J. N. Evolutionary ecology of the relationship between oviposition preference and performance of offspring in phytophagous insects. Entomologia Experimentalis et Applicata, Dordrecht, v. 47, n.1, p. 3-14, 1988.

YAMAMOTO, P. T.; LOPES, S.; BASSANEZI, R. B.; BELASQUE JUNIOR, J.; SPOSITO, M. B. Citros: estrago à vista. Cultivar Hortaliças e Frutas, Pelotas, v. 8, n. 48, p. 22 - 24. 2008. 